\title{
Burden of noninfluenza respiratory viral infections in adults admitted to hospital: analysis of a multiyear Canadian surveillance cohort from 2 centres
}

\author{
Nelson Lee MD, Stephanie Smith MD, Nathan Zelyas MD, Scott Klarenbach MD, Lori Zapernick RN, \\ Christian Bekking MSc, Helen So PhD, Lily Yip MSc, Graham Tipples PhD, Geoff Taylor MD, Samira Mubareka MD
}

Cite as: CMAJ 2021 March 29;193:E439-46. doi: 10.1503/cmaj.201748

\begin{abstract}
BACKGROUND: Data on the outcomes of noninfluenza respiratory virus (NIRV) infections among hospitalized adults are lacking. We aimed to study the burden, severity and outcomes of NIRV infections in this population.
\end{abstract}

METHODS: We analyzed pooled patient data from 2 hospital-based respiratory virus surveillance cohorts in 2 regions of Canada during 3 consecutive seasons (2015/16, 2016/17, 2017/18; $n=2119)$. We included patients aged $\geq 18$ years who developed influenza-like illness or pneumonia and were hospitalized for management. We included patients confirmed positive for $\geq 1$ virus by multiplex polymerase chain reaction assays (respiratory syncytial virus [RSV], human rhinovirus/ enterovirus (hRV), human coronavirus (hCoV), metapneumovirus, parainfluenza virus, adenovirus, influenza viruses). We compared patient characteristics, clinical severity conventional outcomes (e.g., hospital length-of stay, 30-day mortality) and ordinal outcomes (5 levels: discharged, receiving convalescent care, acute ward or intensive care unit [ICU] care and death) for patients with NIRV infections and those with influenza.

RESULTS: Among 2119 adults who were admitted to hospital, 1156 patients (54.6\%) had NIRV infections (hRV 14.9\%, RSV $12.9 \%$, hCoV 8.2\%) and 963 patients $(45.4 \%)$ had influenza $(n=963)$. Patients with NIRVs were younger (mean 66.4 [standard deviation 20.4] yr), and more commonly had immunocompromising conditions (30.3\%) and delay in diagnosis (median 4.0 [interquartile range (IQR) 2.0-7.0] days). Overall, $14.6 \%$
(12.4\%-19.5\%) of NIRV infections were acquired in hospital. Admission to ICU (18.2\%, median 6.0 [IQR 3.0-13.0] d), hospital length-of-stay (median 5.0 [IQR 2.0-10.0] d) and 30-day mortality (8.4\%; RSV 9.5\%, hRV 6.6\%, hCoV 9.2\%) and the ordinal outcomes were similar for patients with NIRV infection and those with influenza. Age $>60$ years, immunocompromised state and hospitalacquired viral infection were associated with worse outcomes. The estimated median cost per acute care admission was $\$ 6000$ (IQR \$2000-\$16000).

INTERPRETATION: The burden of NIRV infection is substantial in adults admitted to hospital and associated outcomes may be as severe as for influenza, suggesting a need to prioritize therapeutics and vaccines for at-risk people.
$\mathbf{T}$ he global burden of lower respiratory tract infections is substantial, leading to many hospital admissions and deaths, especially among young children and older adults. ${ }^{1}$ Respiratory viruses are responsible for almost half of such infections in adults that require in-hospital management; previous studies estimate that $28 \%-62 \%$ are caused by noninfluenza respiratory viruses (NIRVs). ${ }^{2-4}$ With some geographical and seasonal variations, respiratory syncytial virus (RSV), human rhinovirus (hRV) and human coronavirus (hCoV) are among the most frequently identified NIRV infections. ${ }^{1-7}$ Most infected adults develop mild, self-limiting illnesses, but increasing evidence suggest that NIRVs, either alone or with coinfecting bacteria, can result in severe pneumonia and death..$^{8,9}$ For instance, RSV has been shown to cause severe respiratory failure, with fatality rates comparable to or exceeding those observed among adults admitted to hospital with influenza. ${ }^{10-12}$ Data on hRV, hCoV and other NIRVs are more limited, owing to the lack of accurate diagnostics and systematic case-finding approaches. ${ }^{7-9}$ However, with the increasing availability of multiplex polymerase chain reaction (PCR) assays that can simultaneously detect influenza 
and NIRVs, these infections are now readily diagnosed as part of a syndromic approach in patients who present with acute respiratory illnesses. ${ }^{2-5,13,14}$ The burden, clinical significance and impacts of NIRVs on the health care system remain inadequately characterized.

To address this gap, we analyzed the relative frequencies, patient characteristics, location of acquisition (community or hospital), severity and clinical outcomes of patients with NIRV and influenza infections diagnosed by multiplex PCR in a cohort of adults admitted to hospital in 2 large Canadian health care centres during a 3-year surveillance period. The associated health care resource use was also estimated.

\section{Methods}

\section{Study population and data source}

We analyzed individual patient data from 2 hospital-based, respiratory virus surveillance cohorts. We included adults aged 18 years or older who developed symptoms of influenza-like illness or pneumonia, were admitted to hospital for management in Edmonton (University of Alberta Hospital) or Toronto (Sunnybrook Health Sciences Centre) and tested positive for 1 or more influenza or noninfluenza respiratory viruses by multiplex PCR. There were no clinical exclusion criteria. We included data from November to April in 2015/16, 2016/17 and $2017 / 18$. Both sites are university-affiliated, publicly funded, tertiary care hospitals with more than 880 and 1300 beds, respectively. Both study hospitals' Infection Prevention \& Control programs have been conducting prospective surveillance for all respiratory viral infections in adults admitted to hospital since 2014. As part of routine care, inpatients with symptoms of influenza-like illness or pneumonia, regardless of time of admission, are tested for viral causes using a multiplex PCR assay. Positive cases are identified by Infection Prevention \& Control on a daily basis to ensure prompt implementation of appropriate isolation precautions and outbreak detection. ${ }^{15,16}$ Research team members extracted and verified data from surveillance data sets and electronic medical records at each site, using identical variable definitions, which we then combined into a central database for analysis. ${ }^{15,16}$ We removed all identifying patient information from the data sets and analyzed only deidentified data.

\section{Study variables}

We studied the following clinical variables: age and sex; underlying immunocompromising conditions; time of symptom onset; location of infection acquisition; times of hospital admission, discharge or transfer to a convalescent hospital for continuing care; times of intensive care unit (ICU) admission and discharge; requirement for respiratory support; and in-hospital death from any cause within 30 days of admission (for infections that were acquired in the community) and of diagnosis (for all cases). ${ }^{15}$ Patients who were considered immunocompromised included transplant recipients, those on immunosuppressants (including long-term corticosteroids), patients undergoing chemotherapy and patients with neutropenia, active malignancy or acquired immunodeficiency. We classified infections as "hospitalacquired" (i.e., onset of infection $>3 \mathrm{~d}$ postadmission) or "community-acquired."16

We analyzed laboratory variables, including virus type and subtype, specimen type, time of specimen collection and assay turnaround time. We also analyzed data on bacterial or fungal pathogens identified by culture of blood and respiratory samples collected while in hospital (Appendix 1, available at www.cmaj. ca/lookup/doi/10.1503/cmaj.201748/tab-related-content).

\section{Virologic assays}

Clinical samples used for virologic diagnosis included upper (nasal or nasopharyngeal swabs) and lower (bronchoalveolar lavage, bronchial wash or aspirate, endotracheal aspirates) respiratory tract specimens. At both study sites, testing was performed using the Respiratory Viral Panel or the Respiratory Pathogens Panel (Luminex Molecular Diagnostics), which identify influenza $\mathrm{A}$ (with $\mathrm{H} 1_{\text {pdmog }}$ and $\mathrm{H} 3$ subtypes) and $\mathrm{B}$ viruses, respiratory syncytial virus (RSV A and B), parainfluenza viruses (PIV 1-4); human rhinovirus/enterovirus (hRV), human coronaviruses (hCoV HKU1, OC43, NL63, 229E), human metapneumoviruses (hMPV), adenovirus (AdV) and human bocavirus (hBoV). Sample processing, laboratory methods and analytical performances have been described. ${ }^{15-18}$ The assay cannot distinguish between rhinoviruses and enteroviruses; however, available data show that, unlike for children, target detection in adults admitted to hospital rarely represent enteroviruses, especially outside of the summer months. ${ }^{18-20}$ We defined noninfluenza respiratory viruses (NIRVs) as the viral pathogens detected by the multiplex PCR panel (RSV, hRV, hCoV, PIV, hMPV, AdV, hBoV), except the influenza $A$ and $B$ viruses.

\section{Data analysis}

The main analyses compared patient outcomes of NIRV and influenza virus infections. We performed subgroup analyses for the most common viruses, RSV and hRV. Building on our earlier, single-centre study on the burden and significance of hCoV infections, ${ }^{16}$ we also analyzed outcomes of patients with hCoVs in this cohort. Although these coronaviruses are distinct from the pandemic pathogen, severe acute respiratory syndrome coronavirus 2 (SARS-CoV-2), such results may provide a broader perspective on human coronavirus diseases. ${ }^{21,22}$ We reported frequency distribution of patient characteristics. We analyzed conventional severity and outcome indicators, namely requirement for respiratory support, ICU admission, survivors' length-of-stay in hospital and 30-day mortality.

Owing to the diverse clinical trajectories of adults admitted to hospital with respiratory viral infections, we further analyzed outcomes using an ordinal scale method. ${ }^{15,23}$ The scale comprised 5 mutually exclusive clinical status groups, ranked in ascending order of severity: discharged home, receiving continuing care at a convalescent hospital, receiving care in an acute care hospital, receiving ICU care and death. We performed ordinal regression to compare outcomes between patients with NIRV and influenza infections at 30 days after diagnosis. We analyzed potential confounders as covariates in the model, including age, 
immunocompromised state and location of infection acquisition. These variables were found to be significant predictors of clinical status in univariate analyses. We calculated proportional odds ratios (OR) and 95\% confidence intervals $(\mathrm{Cl})$ for each variable. ${ }^{15,23}$ We also performed a secondary analysis to compare patient clinical status at 15 days after diagnosis.

We estimated the health care resource use associated with the index, acute hospital admission because of NIRV or influenza virus infections. We assigned a cost for each unit of resource, including cost-per-day in a medical ward and in the ICU, and calculated the cost of admission for each patient according to the duration of stay in the medical ward or ICU. The unit cost was based on 2016 data from the Canadian Institute of Health Information, inflated to cost in 2018, according to the Canadian Consumer Price Index. ${ }^{24,25}$ The median (interquartile range [IQR]) cost per admission, rounded to the nearest thousand, was reported for the NIRV and influenza patient groups. Both survivors and nonsurvivors were included in the analyses; patients with hospital-acquired viral infections and those with convalescent hospital stays were not included.

\section{Ethics approval}

Ethical approval for data collection was obtained from the institutional review boards of the participating sites.

\section{Results}

Over the 3 study seasons, the 2 hospitals performed more than 7900 tests in adults (in 2015/16, 2016/17 and 2017/18, 1311, 1282 and 1599 tests were performed at site 1 , and 1007, 1163 and 1545 were performed at site 2 , respectively), with overall positivity rates of $27 \%-35 \%$. After excluding duplicate or repeat samples, those positive for bacterial targets and those from nonadmitted patients, we analyzed data from 1014 eligible cases from the Edmonton site and 1105 from the Toronto site. Overall, we analyzed data from 2119 adult inpatients.

The relative frequencies of NIRV infections ( $n=1156,54.6 \%)$ and influenza virus infections ( $n=963,45.4 \%$ ) identified during 3 consecutive seasons are depicted in Figure 1 . Among the former, the most commonly identified viruses were hRV $(n=315$, $14.9 \%)$ and RSV ( $n=274,12.9 \%)$, followed by hMPV $(n=206,9.7 \%)$ and hCoV ( $n=174,8.2 \%)$. Mixed viral infections were identified in $2.0 \%$ of cases ( $n=43 ; 12$ involved a hCoV). The seasonal peak activity observed for influenza virus and RSV infections were consistent with the population surveillance data; this was not apparent for other NIRVs such as hRV (Appendix 1). The baseline characteristics of patients with NIRV and influenza virus infections, as well as their clinical severity and outcomes, are described in Table 1. Patients with NIRV infections were significantly younger

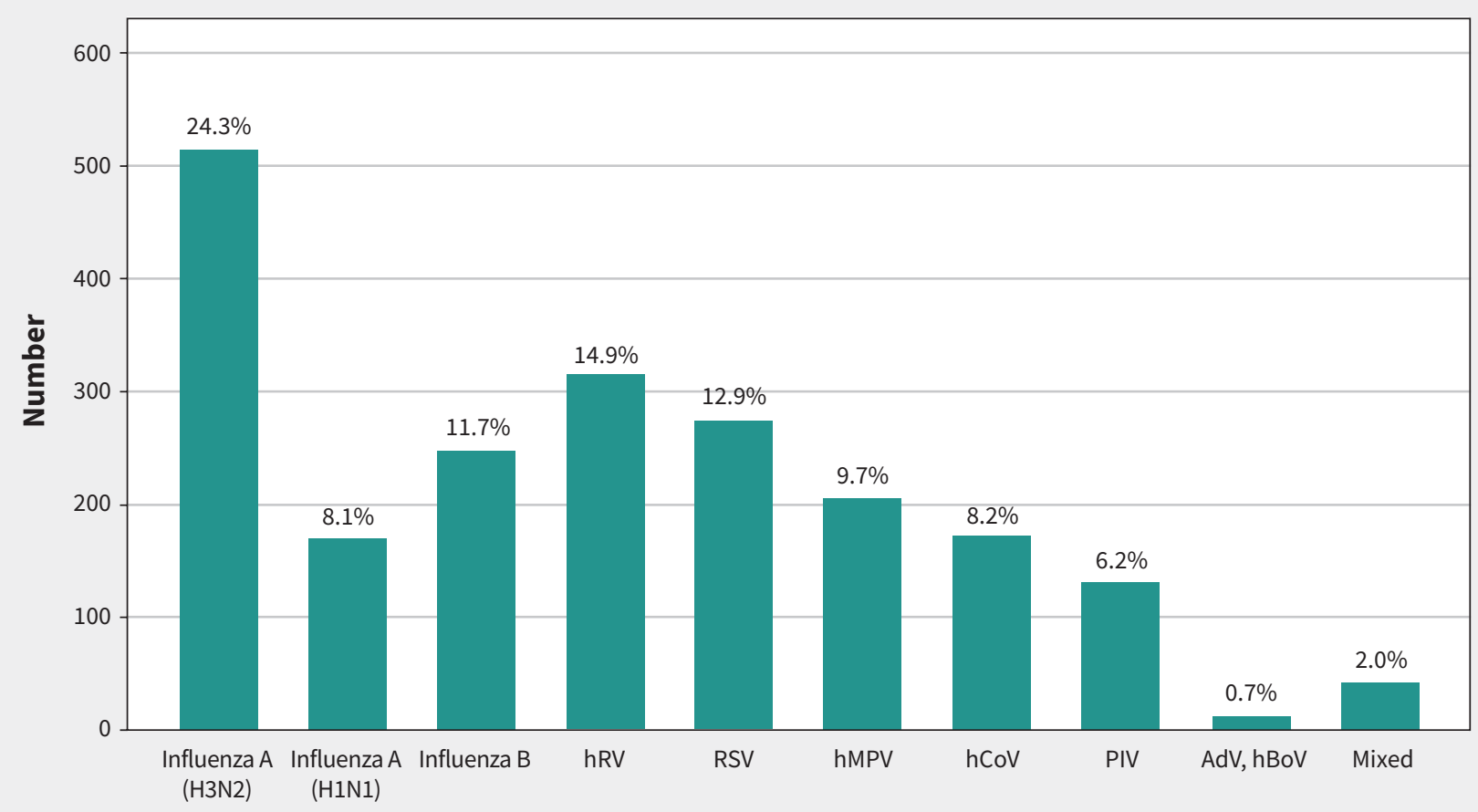

Virus

Figure 1: Frequency distribution of influenza viruses and noninfluenza respiratory viruses (NIRVs) detected in 2119 adults admitted to hospital. Note: The frequency of all influenza viruses was 45.4\%, including influenza A (H3N2), influenza A (H1N1), influenza B and untyped influenza A (not shown, $1.3 \%$ ). The frequency of all NIRVs was $54.6 \%$, including human rhinovirus/enterovirus (hRV), respiratory syncytial virus (RSV), human metapneumovirus (hMPV), human coronavirus (hCoV), parainfluenza viruses (PIV), adenovirus (AdV) or bocavirus (hBoV), and mixed viruses. The identified hCoV strains (including those in mixed infections) were: OC43 $(n=89)$, NL63 $(n=46), 229 \mathrm{E}(n=30)$ and HKU1 $(n=21)$. 
Table 1: Baseline characteristics, severity and outcomes of 2119 adults admitted to hospital with confirmed NIRV and influenza virus infections

\begin{tabular}{|c|c|c|c|c|c|c|}
\hline Variable & $\begin{array}{l}\text { No. }(\%) \text { of } \\
\text { patients with } \\
\text { influenza* } \\
n=963\end{array}$ & $\begin{array}{c}\text { No. }(\%) \text { of } \\
\text { patients with } \\
\text { NIRV* }^{\star} \\
n=1156\end{array}$ & $\begin{array}{c}\text { No. }(\%) \text { of } \\
\text { patients with } \\
\text { RSV* }^{\star} \\
n=\mathbf{2 7 4}\end{array}$ & $\begin{array}{c}\text { No. }(\%) \text { of } \\
\text { patients with } \\
\text { hRV* } \\
n=315\end{array}$ & $\begin{array}{c}\text { No. }(\%) \text { of } \\
\text { patients with } \\
\text { hCoV* } \\
n=174\end{array}$ & $\begin{array}{c}p \\
\text { valuet }\end{array}$ \\
\hline \multicolumn{7}{|l|}{ Age, yr } \\
\hline Mean \pm SD & $68.8 \pm 18.4$ & $66.4 \pm 20.4$ & $69.4 \pm 18.5$ & $61.9 \pm 21.4$ & $66.4 \pm 20.3$ & 0.005 \\
\hline Median (IQR) & $72.0(58.0-84.0)$ & $69.0(54.0-83.0)$ & $72.0(61.0-82.5)$ & $66.0(45.0-80.0)$ & $68.5(55.8-83.3)$ & 0.030 \\
\hline Sex, male & $472(49.1)$ & $559(48.4)$ & $127(46.4)$ & $162(51.4)$ & $83(47.7)$ & 0.728 \\
\hline Immunocompromised $\ddagger$ & $208(25.1)$ & $276(30.3)$ & $64(28.8)$ & $84(34.4)$ & $40(27.4)$ & 0.016 \\
\hline Pregnant femaleł & $21(4.4)$ & $33(5.8)$ & $4(2.8)$ & $12(8.5)$ & $8(4.7)$ & 0.322 \\
\hline $\begin{array}{l}\text { Illness onset to diagnosis, median } \\
\text { (IQR), d§ }\end{array}$ & $3.0(2.0-6.0)$ & $4.0(2.0-7.0)$ & $4.0(2.0-7.0)$ & $3.0(2.0-7.0)$ & $3.0(2.0-7.0)$ & 0.016 \\
\hline LRT samples for diagnosis§ & $45(4.7)$ & $69(6.0)$ & $3(1.1)$ & $19(6.1)$ & $8(4.6)$ & 0.187 \\
\hline Hospital-acquired infection§ & $131(13.6)$ & $169(14.6)$ & $34(12.4)$ & $41(13.0)$ & 34 (19.5) & 0.504 \\
\hline Respiratory supportø & $181(18.8)$ & $243(21.1)$ & $56(20.7)$ & $60(19.2)$ & $34(19.5)$ & 0.182 \\
\hline ICU admission & $159(16.5)$ & $210(18.2)$ & $50(18.2)$ & $50(15.9)$ & $30(17.2)$ & 0.317 \\
\hline ICU length-of-stay, median (IQR), d & $6.0(3.0-13.3)$ & $6.0(3.0-13.0)$ & $6.0(3.0-9.0)$ & $7.0(2.5-14.5)$ & $5.0(2.8-8.3)$ & 0.902 \\
\hline \multicolumn{7}{|l|}{ Bacterial isolates ${ }^{\star \star}$} \\
\hline Blood samples & $19(3.7)$ & $52(4.5)$ & $11(4.0)$ & $17(5.4)$ & $9(5.2)$ & 0.443 \\
\hline Respiratory samples & $22(4.3)$ & $256(22.2)$ & $46(16.8)$ & $74(23.5)$ & $30(17.2)$ & $<0.001$ \\
\hline $\begin{array}{l}\text { Hospital length-of-stay, median } \\
\text { (IQR), d†† }\end{array}$ & $4.0(2.0-9.0)$ & $5.0(2.0-10.0)$ & $5.0(3.0-9.0)$ & $4.0(2.0-8.0)$ & $4.0(2.0-10.0)$ & 0.041 \\
\hline 30-day mortality from admission $\dagger \dagger$ & $45(5.4)$ & $77(7.8)$ & $20(8.3)$ & $19(6.9)$ & $11(7.9)$ & 0.043 \\
\hline 30-day mortality from diagnosis $† \dagger$ & $62(6.5)$ & $98(8.4)$ & $26(9.5)$ & $21(6.6)$ & $16(9.2)$ & 0.077 \\
\hline
\end{tabular}

Note: $\mathrm{hCoV}=$ human coronavirus, $\mathrm{hRV}=$ human rhinovirus, ICU = intensive care unit, IQR = interquartile range, $\mathrm{LRT}=$ lower respiratory tract, $\mathrm{NIRV}=$ noninfluenza respiratory virus,

RSV = respiratory syncytial virus, $S D=$ standard deviation

*Unless indicated otherwise.

tComparison between all NIRV and influenza patients ( $\chi^{2}$ test, Mann-Whitney $U$ test and Student $t$ test were used, whenever appropriate).

fImmunocompromised patients $(n=1738$ ) included recipients of transplant, patients on immunosuppressants (including long-term corticosteroids), patients undergoing

chemotherapy and patients with neutropenia, active malignancy or acquired immunodeficiency. Pregnant female reflects the frequency among female subjects only.

§lllness onset to diagnosis is defined as the time between illness onset and diagnostic specimen collection; the onset date was undetermined in about $23.8 \%$ of cases. The median

time interval between admission and diagnostic specimen collection for patients with hospital-acquired infections was 12 (IQR 4-24) days. Lower respiratory tract (LRT) includes

patients for whom samples were required to establish the diagnosis, which included bronchoalveolar lavage, bronchial wash or aspirate and endotracheal aspirates.

IRespiratory support includes patients who required intubation and mechanical ventilation, noninvasive ventilation (e.g., bilevel positive airway pressure), high-flow oxygen therapy (HFOT) and extracorporeal membrane oxygenation (ECMO). Data on supplemental oxygen use was available from 1 centre ( $n=628$; influenza $68.0 \%$, NIRV $63.6 \%, p>0.05$ ).

${ }^{\star \star}$ Bacterial isolates includes cases with $\geq 1$ bacterial pathogens identified in cultures of blood or respiratory samples (Appendix 2).

††Hospital length-of-stay was calculated for survivors with community-acquired infections $(n=1681)$. Thirty-day mortality from admission includes only patients with communityacquired infections $(n=1817)$. Thirty-day mortality from diagnosis includes all 2119 patients. Thirty-day mortality of patients admitted to the ICU was $16.4 \%$ and $21.0 \%$ among influenza $(n=159)$ and NIRV ( $n=210)$ patients, respectively (92\% of ICU admissions occurred on day $1-2$ of hospitalization); mortality of non-ICU cases was $4.5 \%$ and $5.7 \%$, respectively.

(mean 66.4 [standard deviation (SD) 20.4] yr v. 68.8 [SD 18.4] yr), more frequently had underlying immunocompromising conditions (30.3\% v. $25.1 \%)$ and more often experienced delay in diagnosis (median 4.0 [IQR 2.0-7.0] d v. 3.0 [IQR 2.0-6.0] d from illness onset) than patients with influenza. Overall, $14.6 \%$ of NIRV infections were acquired in hospital (RSV 12.4\%, hRV 13.0\%, hCoV $19.5 \%$; hCoV v. influenza, $p=0.047$ ). The median time interval between admission and diagnosis among these patients was 12 (IQR 4-24) days.

A substantial proportion of patients with NIRV infections required respiratory support $(21.1 \%)$ and ICU care (18.2\%), with a median ICU stay of 6.0 (IQR 3.0-13.0) days. In 22.2\% of patients with NIRV infections, 1 or more bacterial or fungal pathogens were isolated from respiratory samples (Appendix 2, available at www. cmaj.ca/lookup/doi/10.1503/cmaj.201748/tab-related-content).
For the entire cohort, including both community- and hospitalacquired NIRV infections, the 30-day mortality from time of diagnosis was $8.4 \%$ (RSV 9.5\%, hRV 6.6\%, hCoV 9.2\%). The 30-day mortality from time of admission was $7.8 \%$ for patients with community-acquired NIRV infections. The median hospital length of stay among survivors was 5.0 (IQR 2.0-10.0) days. These results were generally comparable to those observed among patients with influenza (Table 1). Kaplan-Meier analyses showed no overall difference in survival between patients with NIRV infections and those with influenza (Figure $2 \mathrm{~A}$ and $2 \mathrm{~B}$ ). A subgroup analysis showed a trend toward lower survival in patients with hCoV infections, compared with influenza A (log-rank test, $p=0.096$ ).

Change in clinical status among patients with NIRV and influenza virus infections over a 30-day period is depicted in Figure 3. At 15 days after diagnosis, $71.4 \%$ of patients with NIRV infections were 
A) All NIRV v. IV infections (log-rank, $p=0.076$ )

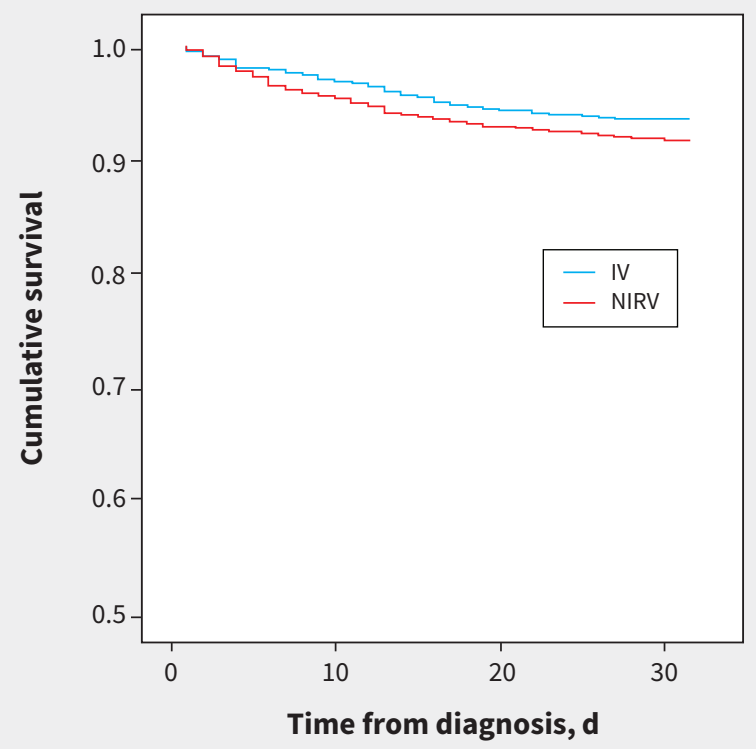

B) Specific NIRV v. IV infections (log-rank, $p=0.248$ )

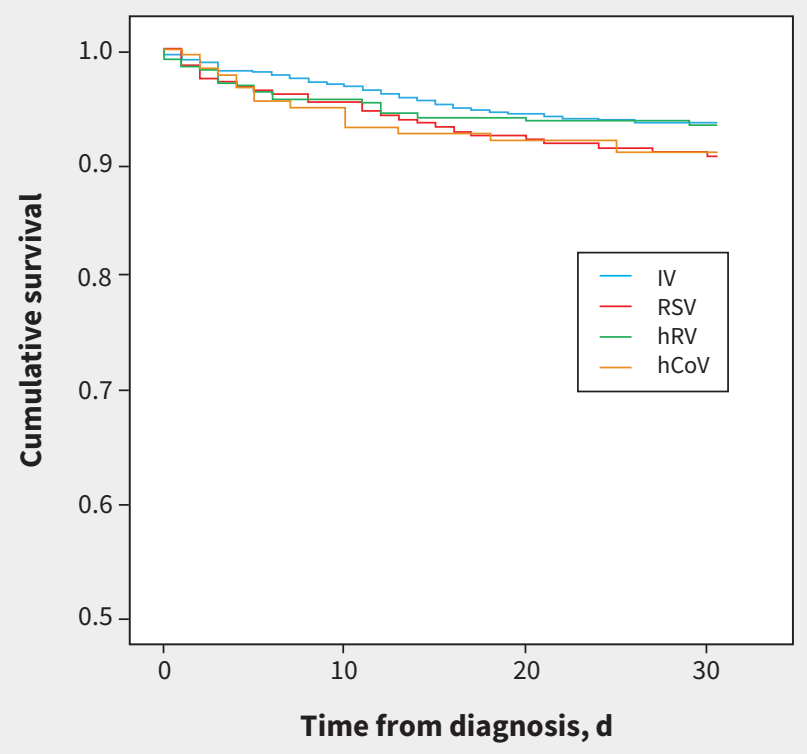

Figure 2: Kaplan-Meier survival analysis in adults admitted to hospital with (A) all noninfluenza respiratory virus (NIRV) infections and (B) specific NIRV infections, compared with influenza virus (IV) infections. Note: hRV = human rhinovirus/enterovirus, RSV = respiratory syncytial virus, hCoV= human coronavirus.

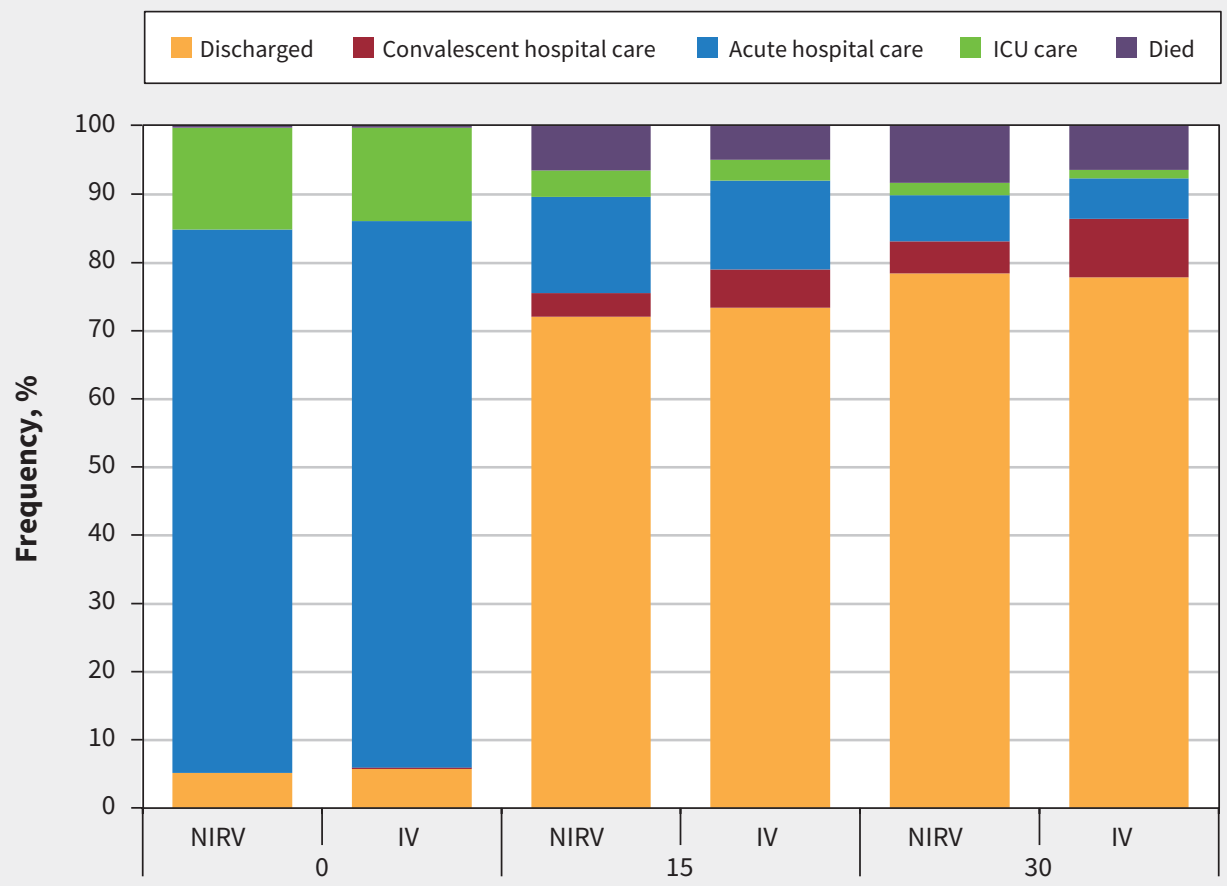

Time from diagnosis, $\mathrm{d}$

Figure 3: Ordinal outcome (clinical status) changes over 30 days since diagnosis (at baseline, day 15 and day 30) among adults inpatients with noninfluenza respiratory virus (NIRV) and influenza virus (IV) infections. Note: ICU = intensive care unit. 
Table 2: Ordinal outcomes of 2119 adult inpatients with NIRV and influenza infections*

No. of

patients with

clinical status

available

No. (\%)

Infection

discharged

\author{
No. $(\%)$ in
}

convalescent

hospital care

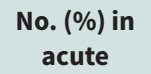

hospital care

No. $(\%)$
in ICU

care

No.

(\%)

died

$p$ value

At $15 \mathrm{~d}$

Influenza

962

1154

273

314

524

711 (73.9)

824 (71.4)

$52(5.4)$

41 (3.6)

198 (72.5)

230 (73.2)

Other NIRVsł

$368(70.2)$

6 (2.2)

9 (2.9)

$26(5.0)$

122 (12.7)

166 (14.4)

40 (14.7)

$29(3.0)$

$48(5.0)$

47 (4.1)

76 (6.6)

0.034

44 (14.0)

72 (13.7)

$10(3.7)$

$19(7.0)$

0.058

$12(3.8)$

19 (6.1)

0.256

At $30 \mathrm{~d}$

\begin{tabular}{cc} 
Influenza & 96 \\
NIRV & 1154 \\
RSV & 273 \\
hRV & 31 \\
\hline Other NIRVst & 524
\end{tabular}

Other NIRVs
961

1154

273

314

524

$\begin{array}{lc}753(78.4) & 80(8.3) \\ 898(77.8) & 56(4.9) \\ 215(78.8) & 9(3.3) \\ 257(81.8) & 11(3.5) \\ 395(75.4) & 33(6.3)\end{array}$

55 (5.7)

$82(7.1)$

$19(7.0)$

$19(6.1)$

$38(7.3)$
22 (4.2)

$36(6.9)$

0.151

Note: hRV = human rhinovirus, ICU = intensive care unit, NIRV = noninfluenza respiratory virus, RSV = respiratory syncytial virus.

*Ordinal regression was performed to compare the 5 -level ordinal outcomes at 15 days and 30 days since diagnosis. Age, immunocompromised state and location of infection acquisition (shown to be significant in univariate analyses) were included as covariates in each model. Analyses limited to community-acquired

infections showed no significant difference between virus groups; the variable "sex" was also insignificant (data not shown). For hCoV, the ordinal outcomes at

30 days were as follows: $81.6 \%$ discharged, $3.4 \%$ in convalescent hospital care, $4.6 \%$ in acute hospital care, $1.1 \%$ in ICU care and $9.2 \%$ died.

tCompared with influenza.

†Other NIRVS include human metapneumovirus, human coronavirus, parainfluenza viruses, adenovirus or bocavirus.

discharged and 3.6\% were receiving continuing care at a convalescent hospital; $18.5 \%$ were still receiving care in acute care wards or in ICUs, and $6.6 \%$ had died. At 30-days after diagnosis, the proportions were $77.8 \%, 4.9 \%, 8.9 \%$ and $8.4 \%$, respectively. The changes were comparable to those observed for patients with influenza. Ordinal regression showed that the outcomes of patients with NIRV infections 30 days after diagnosis were not significantly different from those with influenza (OR 1.17, 95\% Cl 0.93-1.48) (Table 2). Immunocompromised state (OR 1.40, 95\% Cl 1.08-1.82), hospitalacquired infection (OR $3.49,95 \% \mathrm{Cl} 2.65-4.61$ ) and older age (61-80 yr: OR 1.63, 95\% Cl 1.04-2.55; > 80 yr: OR 2.22, 95\% Cl 1.423.48 , compared with younger age groups $<60 \mathrm{yr}$ ) predicted worse clinical outcomes for both patient groups in the final model. We found the clinical status of patients with NIRV infections at 15 days postdiagnosis to be slightly worse than that of patients with influenza (OR 1.27, 95\% Cl 1.02-1.57; Table 2).

The overall median cost for patients admitted to hospital with acute NIRV or influenza infections was estimated to be $\$ 6000$ (IQR $\$ 2000-\$ 15000)$ per acute care hospital admission. Patients admitted to ICU and non-ICU wards cost $\$ 29000$ (\$17000-\$58000) and \$5000 (\$2000-\$10000), respectively. There was no significant difference in costs accrued by patients with NIRV (\$6000 [IQR \$2000-\$16000]) and influenza (\$6000 [IQR \$2000-\$13000]) infections (Appendix 3, available at www.cmaj.ca/lookup/doi/10.1503/cmaj.201748/tab-related -content). In the participating hospitals, as about 300 patients (NIRV and influenza infections combined) were admitted during each seasonal peak, the estimated median health care cost attributable to hospital admissions for viral lower respiratory tract infections was around \$1814000 (IQR \$725000-\$4352000) per hospital per season.

\section{Interpretation}

In this two-centre, multiseason cohort of adults admitted to hospital with laboratory-confirmed viral acute respiratory tract infections, NIRV infections were more frequently (54.6\%) identified than influenza viruses (45.4\%). Respiratory failure necessitating ventilatory support developed in about one-fifth (21.1\%) of patients with NIRV infections; one-quarter required prolonged hospital stay > 10 days (median 5, IQR 2-10 d); and 8.4\% (6.4\%$9.5 \%$ ) died within 30 days of diagnosis. These findings show that clinical status changes for patients with NIRV infections were comparable to patients with influenza. The associated costs of hospital admission were substantial.

The strengths of our study include its 2-centre, multiseason design, collective analyses of NIRVs, large sample size and comprehensive outcome evaluation using conventional and ordinal scale methods, with influenza as a comparator. Consistent with other studies using a syndromic testing approach, we found that, collectively, NIRVs are at least as common as influenza in adults admitted to hospital for viral respiratory infections, suggesting substantial disease burden in this unique population. ${ }^{2-4,8,9} \mathrm{We}$ also found that the clinical course and outcomes of patients admitted to hospital with NIRV infections are generally comparable to those of patients with influenza, leading to substantial morbidity and deaths. ${ }^{10-12,26-32}$ Patients with NIRV infections were younger, but more commonly had underlying conditions. Host factors, including immunocompromised state, advanced age and infection acquired during hospital care for other conditions (i.e., comorbidities), independently predicted adverse outcomes. 
Unlike for influenza, effective vaccines and antiviral treatment or prophylaxis are currently unavailable for these patients at high risk of severe NIRV infection, which, alongside other emerging data highlights an important research gap. ${ }^{1,7-10,33}$ Notably, a substantial proportion (15\%-20\%) of NIRV infections were found to be acquired in hospital. The initial mild symptoms, insidious onset and progression ${ }^{10,28,29}$ and the long delay to receive virologic test results in clinical practice (1-2 d; Appendix 4, available at www.cmaj.ca/lookup/doi/10.1503/cmaj.201748/tab-related -content), ${ }^{13,14}$ are barriers to case identification and prompt isolation. Multimodal infection control bundles to address potential transmission routes and strategies for rapid case detection should be further optimized. ${ }^{3,11,13,13,34,35}$

The most frequently identified NIRVs were RSV and rhinoviruses. ${ }^{1-7}$ Respiratory syncytial virus can cause pneumonia, acute bronchitis or bronchiolitis and acute exacerbation of underlying airway disease in adults, leading to respiratory failure. ${ }^{8-13}$ Similar to a recent report, ${ }^{12}$ we observed trends toward worse clinical status and higher fatality in patients with RSV than among those with influenza. For rhinoviruses, emerging evidence from pathogenesis and clinical studies suggest their roles in acute exacerbation of airway diseases and pneumonia in immunocompromised, as well as immunocompetent adults. ${ }^{8,9,29,30}$ Our data further showed an association of rhinovirus infections with adverse outcomes comparable to influenza. Human metapneumovirus and coronaviruses are the other NIRVs most frequently identified in adults admitted to hospital, as reviewed elsewhere. , $9,27,36^{\text {Limited }}$ data on metapneumovirus described wheezing, dyspnea and pneumonia, frequently without fever, in older adults with comorbidities, as well as institutional outbreaks. ${ }^{8,9,31}$ In this study, $16 \%$ of NIRV infections were hCoVs (including OC43, NL63, 229E and HKU1, in descending frequency); $19.5 \%$ of patients with hCoV infections developed respiratory failure, with a trend toward higher fatality than influenza $(9.2 \%$ v. $6.5 \%)$. These findings are consistent with the findings of our previous single-centre study (ICU admission 17\%, fatality 7\%), ${ }^{16}$ and a smaller study on patients admitted to hospital with hCoV $(n=29$; dyspnea $45 \%$, ICU admission 31\%). ${ }^{36}$ Moreover, almost $20 \%$ of hCoV infections were hospital-acquired, a higher rate than for other viruses. Data on hospital transmission are few,, 28 but substantial household secondary attack rates of hCoV NL63 (12.6\%) and OC43 (10.6\%) have recently been described. ${ }^{22}$ Further research on hCoV transmission and its pathogenic behaviour in susceptible people is necessary. ${ }^{9,26-28}$

Data on the economic burden and health care cost of NIRV infections is limited compared with influenza. ${ }^{37-40}$ We found that the median cost of an admission to hospital for NIRV infection is around $\$ 6000$ per admission (IQR \$2000-\$16000), comparable to the cost for an influenza admission. The cost of 150 NIRV-related admissions in each season would be nearly 1 million dollars, which is likely an underestimation as we are unable to include detailed expenditures (e.g., investigations, medications), convalescent care and hospital-acquired viral infections in the calculation, limited by study design. A more comprehensive, prospective evaluation has been planned to better inform future health care planning.

\section{Limitations}

We were unable to address the relative risks for admission to hospital with NIRVs. Despite our testing efforts, ${ }^{2-4}$ it remains possible that some cases of lower respiratory tract viral infectionwere undetected if a nonrespiratory condition was the cause of admission. ${ }^{13,14}$ Our surveillance programs did not include interseasonal periods (May-October); nevertheless, current data show that RSV and hCoV, among other viruses (except hRV), generally follow a seasonal pattern in temperate climates. ${ }^{10,22,29,32}$ The role of underlying comorbidities and the pathophysiology of severe manifestations warrant further study. We reported high frequencies of bacterial and fungal pathogens, isolated from respiratory samples of patients with NIRV infections (22\% v. 4\%; Table 1; Appendix 2) suggesting secondary infections, but further evaluation was limited by our study design. ${ }^{10,11}$ Our results, while likely generalizable to larger, regional acute care centres, are not generalizable to pediatric populations, longer-term care centres or rural health care settings.

\section{Conclusion}

NIRV infections account for a substantial proportion of the disease burden among adults admitted to hospital with respiratory tract infections. They are associated with severe clinical outcomes, similar to influenza. Our findings highlight unmet needs and research gaps in therapeutics and vaccines for people at high risk of NIRV infection.

\section{References}

1. GBD 2016 Lower Respiratory Infections Collaborators; Estimates of the global, regional, and national morbidity, mortality, and aetiologies of lower respiratory infections in 195 countries, 1990-2016: a systematic analysis for the Global Burden of Disease Study 2016. Lancet Infect Dis 2018;18:1191-210.

2. Jain S, Self WH, Wunderink RG, et al.; CDC EPIC Study Team. Communityacquired pneumonia requiring hospitalization among U.S. adults. N Engl J Med 2015;373:415-27.

3. Brendish NJ, Malachira AK, Armstrong L, et al. Routine molecular point-of-care testing for respiratory viruses in adults presenting to hospital with acute respiratory illness (ResPOC): a pragmatic, open-label, randomised controlled trial. Lancet Respir Med 2017;5:401-11.

4. Zhou F, Wang Y, Liu Y, et al.; CAP-China Network. Disease severity and clinical outcomes of community-acquired pneumonia caused by non-influenza respiratory viruses in adults: a multicentre prospective registry study from the CAPChina Network. Eur Respir J 2019;54:1802406.

5. leven M, Coenen S, Loens K, et al.; GRACE consortium. Aetiology of lower respiratory tract infection in adults in primary care: a prospective study in 11 European countries. Clin Microbiol Infect 2018;24:1158-63.

6. Shi T, Arnott A, Semogas I, et al.; RESCEU Investigators. The etiological role of common respiratory viruses in acute respiratory infections in older adults: a systematic review and meta-analysis. J Infect Dis 2020;222(Suppl 7):S563-9.

7. Tang JW, Lam TT, Zaraket H, et al.; INSPIRE investigators. Global epidemiology of non-influenza RNA respiratory viruses: data gaps and a growing need for surveillance. Lancet Infect Dis 2017;17:e320-6.

8. Walter JM, Wunderink RG. Severe respiratory viral infections: new evidence and changing paradigms. Infect Dis Clin North Am 2017;31:455-74.

9. Kodama F, Nace DA, Jump RLP. Respiratory syncytial virus and other noninfluenza respiratory viruses in older adults. Infect Dis Clin North Am 2017;31:767-90.

10. Griffiths C, Drews SJ, Marchant DJ. Respiratory syncytial virus: infection, detection, and new options for prevention and treatment. Clin Microbiol Rev 2017;30:277-319.

11. Lee N, Lui GCY, Wong KT, et al. High morbidity and mortality in adults hospitalized for respiratory syncytial virus infections. Clin Infect Dis 2013;57:1069-77.

12. Ackerson B, Tseng HF, Sy LS, et al. Severe morbidity and mortality associated with respiratory syncytial virus versus influenza infection in hospitalized older adults. Clin Infect Dis 2019;69:197-203. 
13. Lee N, Walsh EE, Sander I, et al. Delayed diagnosis of respiratory syncytial virus infections in hospitalized adults: individual patient data, record review analysis and physician survey in the United States. J Infect Dis 2019;220:969-79.

14. Drews SJ, Branche AR, Falsey AR, et al. What is the role of rapid molecular testing for seniors and other at-risk adults with respiratory syncytial virus infections? J Clin Virol 2019;117:27-32.

15. Lee N, Smith SW, Hui DSC, et al. Development of an ordinal scale treatment endpoint in adults hospitalized with influenza. Clin Infect Dis 2020 June 17 [Epub ahead of print]. doi: 10.1093/cid/ciaa777.

16. Kozak R, Prost K, Yip L, et al. Severity of coronavirus respiratory tract infections in adults admitted to acute care in Toronto, Ontario. J Clin Virol 2020;126:104338.

17. Fathima S, Lee BE, May-Hadford J, et al. Use of an innovative web-based laboratory surveillance platform to analyze mixed infections between human metapneumovirus (hMPV) and other respiratory viruses circulating in Alberta (AB), Canada (2009-2012). Viruses 2012;4:2754-65.

18. Gonsalves S, Mahony J, Rao A, et al. Multiplexed detection and identification of respiratory pathogens using the NxTAG ${ }^{\circledR}$ respiratory pathogen panel. Methods 2019;158:61-8.

19. Ko FW-S, Chan PK-S, Chan RWY, et al. Molecular detection of respiratory pathogens and typing of human rhinovirus of adults hospitalized for exacerbation of asthma and chronic obstructive pulmonary disease. Respir Res 2019;20:210.

20. Sundell N, Andersson L-M, Brittain-Long R, et al. PCR detection of respiratory pathogens in asymptomatic and symptomatic adults. J Clin Microbiol 2019;57: e00716-8.

21. Coronavirus disease (COVID-19) pandemic. Geneva: World Health Organization. Available: www.who.int/emergencies/diseases/novel-coronavirus-2019 (accessed 2021 Jan. 15).

22. Monto AS, DeJonge PM, Callear AP, et al. Coronavirus occurrence and transmission over 8 years in the HIVE cohort of households in Michigan. J Infect Dis 2020;222:9-16.

23. King JC, Beigel JH, Ison MG, et al. Clinical development of therapeutic agents for hospitalized patients with influenza: challenges and innovations. Open Forum Infect Dis 2019;6:ofz137.

24. Care in Canadian ICUs. Ottawa: Canadian Institute for Health Information; 2016.

25. Table 18-10-0005-01: Consumer Price Index, annual average, not seasonally adjusted. Ottawa: Statistics Canada. Available: www150.statcan.gc.ca/t1/tbl1/ en/tv.action?pid=1810000501 (accessed 2020 Sept. 15).

26. Corman VM, Muth D, Niemeyer D, et al. Hosts and sources of endemic human coronaviruses. Adv Virus Res 2018;100:163-88.
27. Ogimi C, Waghmare AA, Kuypers JM, et al. Clinical significance of human coronavirus in bronchoalveolar lavage samples from hematopoietic cell transplant recipients and patients with hematologic malignancies. Clin Infect Dis 2017;64:1532-9.

28. Hand J, Rose EB, Salinas A, et al. Severe respiratory illness outbreak associated with human coronavirus NL63 in a long-term care facility. Emerg Infect Dis 2018;24:1964-6

29. Jacobs SE, Lamson DM, St George K, et al. Human rhinoviruses. Clin Microbiol Rev 2013;26:135-62.

30. Miller EK, Linder J, Kraft D, et al. Hospitalizations and outpatient visits for rhinovirus-associated acute respiratory illness in adults. J Allergy Clin Immunol 2016;137:734-43.e1.

31. Widmer K, Griffin MR, Zhu Y, et al. Respiratory syncytial virus- and human metapneumovirus-associated emergency department and hospital burden in adults. Influenza Other Respir Viruses 2014;8:347-52.

32. Russell E, Yang A, Tardrew S, et al. Parainfluenza virus in hospitalized adults: a 7-year retrospective study. Clin Infect Dis 2019;68:298-305.

33. Brendish NJ, Clark TW. Antiviral treatment of severe non-influenza respiratory virus infection. Curr Opin Infect Dis 2017;30:573-8.

34. Taylor G, Mitchell R, McGeer A, et al.; Canadian Nosocomial Infection Surveillance Program. Healthcare-associated influenza in Canadian hospitals from 2006 to 2012. Infect Control Hosp Epidemiol 2014;35:169-75.

35. Blanco N, Eisenberg MC, Stillwell T, et al. What transmission precautions best control influenza spread in a hospital? Am J Epidemiol 2016;183:1045-54.

36. Garbino J, Crespo S, Aubert J-D, et al. A prospective hospital-based study of the clinical impact of non-severe acute respiratory syndrome (Non-SARS)related human coronavirus infection. Clin Infect Dis 2006;43:1009-15.

37. Putri WCWS, Muscatello DJ, Stockwell MS, et al. Economic burden of seasonal influenza in the United States. Vaccine 2018;36:3960-6.

38. Fendrick AM, Monto AS, Nightengale B, et al. The economic burden of noninfluenza-related viral respiratory tract infection in the United States. Arch Intern Med 2003;163:487-94.

39. Amand C, Tong S, Kieffer A, et al. Healthcare resource use and economic burden attributable to respiratory syncytial virus in the United States: a claims database analysis. BMC Health Serv Res 2018;18:294.

40. Ackerson B, An J, Sy LS, et al. Cost of hospitalization associated with respiratory syncytial virus infection versus influenza infection in hospitalized older adults. J Infect Dis 2020;222:962-6.
Competing interests: Nelson Lee declares honoraria for consultancy work, speaking in educational programs or travel support from Shionogi, Gilead Sciences Canada, Janssen Pharmaceuticals, Sanofi-Pasteur, F. HoffmannLa Roche, Genentech, CIDARA Therapeutics, outside the submitted work. Scott Klarenbach is supported by the Kidney Health Research Chair, and the Division of Nephrology at the University of Alberta, outside the submitted work.

This article has been peer reviewed.

Affiliations: Division of Infectious Disease (Lee, Smith, Zapernick, Taylor), Department of Medicine, and Department of Laboratory Medicine and Pathology (Zelyas, Tipples), and Division of Nephrology (Klarenbach, So), Department of Medicine, University of Alberta, Edmonton, Alta.; Sunnybrook Research Institute (Bekking, Yip, Mubareka); Department of Laboratory Medicine and Pathobiology (Mubareka), University of Toronto, Toronto, Ont.
Contributors: Nelson Lee, Stephanie Smith and Samira Mubareka are responsible for conception and design of this work. Nelson Lee, Stephanie Smith, Samira Mubareka, Nathan Zelyas, Lori Zapernick, Christian Bekking, Lily Yip, Graham Tipples and Geoff Taylor contributed to the acquisition of data. Nelson Lee, Stephanie Smith, Samira Mubareka, Scott Klarenbach and Helen So contributed to analysis and interpretation of data. Nelson Lee, Samira Mubareka drafted the manuscript. All of the authors revised it critically for important intellectual content, gave final approval of the version to be published and agreed to be accountable for all aspects of the work.

Content licence: This is an Open Access article distributed in accordance with the terms of the Creative Commons Attribution (CC BY-NC-ND 4.0) licence, which permits use, distribution and reproduction in any medium, provided that the original publication is properly cited, the use is noncommercial (i.e., research or educational use), and no modifications or adaptations are made. See: https://creativecommons.org/ licenses/by-nc-nd/4.0/

Data sharing: If approved by the study hospitals through data sharing agreements, unidentified data can be made available for use by other researchers.

Acknowledgement: The authors thank Dr. Matthew Croxen from the Alberta Provincial Laboratory, Alberta, for his inputs to the manuscript.

Disclaimer: Dr. Geoff Taylor sadly passed away after completion of this work. He was the founder of infection prevention and control surveillance program at the University of Alberta Hospital. His contributions are deeply missed by the Infectious Diseases community.

Accepted: Jan. 20, 2021

Correspondence to: Samira Mubareka, samira.mubareka@sunnybrook.ca; Nelson Lee, laishunn@ualberta.ca 